\title{
Discretization of Electric Current Volume Integral Equation With Piecewise Linear Basis Functions
}

\section{Markkanen, Johannes}

2014-09

Markkanen , J \& Ylä-Oijala , P 2014 , ' Discretization of Electric Current Volume Integral Equation With Piecewise Linear Basis Functions ' , IEEE Transactions on Antennas and Propagation, vol. 62 , no. 9 , pp. 4877-4880 . https://doi.org/10.1109/TAP.2014.2334705

http://hdl.handle.net/10138/136278

https://doi.org/10.1109/TAP.2014.2334705

acceptedVersion

Downloaded from Helda, University of Helsinki institutional repository.

This is an electronic reprint of the original article.

This reprint may differ from the original in pagination and typographic detail.

Please cite the original version. 


\section{Discretization of Electric Current Volume Integral Equation with Piecewise Linear Basis Functions}

\author{
Johannes Markkanen, and Pasi Ylä-Oijala
}

\begin{abstract}
The electric current volume integral equation formulation is discretized with piecewise linear basis and testing functions. The resulting weak formulation is more complicated, and contains additional terms compared with the previous discretization utilizing piecewise constant approximations. However, the use of fully linear functions provides superior solution accuracy while keeping good convergence properties of the original integral equation.
\end{abstract}

Index Terms-Electromagnetic scattering, method of moments, volume integral equation

\section{INTRODUCTION}

Electromagnetic wave scattering by arbitrarily shaped threedimensional inhomogeneous dielectric objects located in free space can be described by the volume integral equations. A numerical solution for the volume integral equation is most often found through the method of moments in which the scatterer is divided into simple elements, and the basis functions associated with these elements are used for expanding the unknown function. Then, the testing procedure, such as the Galerkin, Petrov-Galerkin, collocation, etc, is applied to convert the continuous equation into a system of linear equations which can be solved numerically. The accuracy of this technique is highly dependent on the choice of the basis and testing functions as well as the integral equation formulation applied.

Several different types of basis and testing functions have been proposed in the past. The most commonly used basis functions are known as the SWG (Schaubert-Wilton-Glisson) basis functions [1]. These functions are divergence conforming, i.e., they force the normal continuity, and therefore, are used to expand the electric flux density. The SWG functions, however, do not generally satisfy the divergence free condition that may spoil the convergence of the solution [2]. Remedy for this, the purely solenoidal basis functions were proposed [3][5], but the use of these functions deteriorates the convergence of the iterative solution. It is also possible to write the integral equation for the electric field with the curl conforming functions [6]-[9], as well as for the polarization current with discontinuous or $L^{2}$-conforming [10]-[11] functions. Moreover, the potential formulations with continuous basis functions were proposed in [12], [13]. However, the accuracies of these potential formulations are not satisfactory due to the point-matching testing scheme applied.

Manuscript received yyy; revised zzz.

The research has been funded by the ERC Advanced Grant No 320773 entitled Scattering and Absorption of Electromagnetic Waves in Particulate Media (SAEMPL).

J. Markkanen is with the Department of Physics, University of Helsinki e-mail: johannes.markkanen@helsinki.fi

P. Ylä-Oijala is with the Department of Radio Science and Engineering, Aalto University
The choice of the testing functions is more complicated than the choice of the basis functions, especially, if the $L^{2}$ inner product is applied. In which case, the testing functions should span the $L^{2}$-dual space of the range of the operator, and constructing a proper set of functions can be untrivial. Hence, working fully in $L^{2}$ should give rise to a simple properly discretized system as proposed in [14].

In [11], the electric and magnetic current volume integral equation formulation, which maps from $L^{2}$ to itself, was discretized with piecewise constant functions. However, it is clear that the piecewise constant approximations require very small elements to be able to provide an accurate representation for the unknown function. Here, in order to improve the accuracy of the electric current (J-VIE), the equation is discretized with linear basis and testing functions.

\section{J-VIE FORMULATION}

Consider electromagnetic wave scattering by a linear inhomogeneous three-dimensional object bounded by a domain $\Omega$ in homogeneous background medium with constants $\epsilon_{0}$ and $\mu_{0}$. The total time-harmonic $\left(e^{-i \omega t}\right)$ electric field $\boldsymbol{E}$ due to the equivalent volume polarization current is given by [15]

$$
\boldsymbol{E}=\boldsymbol{E}^{\mathrm{inc}}+\frac{-1}{i \omega \epsilon_{0}}\left(\nabla \nabla+k_{0}^{2} \overline{\bar{I}}\right) \cdot \mathcal{V}(\boldsymbol{J}),
$$

where $\boldsymbol{E}^{i n c}$ is the incident electric field, and $k_{0}=\omega \sqrt{\epsilon_{0} \mu_{0}}$ is the wavenumber in the background medium. The equivalent electric polarization current is defined as

$$
\boldsymbol{J}(\boldsymbol{r})=-i \omega \varepsilon_{0}\left(\overline{\bar{\epsilon}}_{r}(\boldsymbol{r})-\overline{\bar{I}}\right) \cdot \boldsymbol{E}(\boldsymbol{r}),
$$

in which $\overline{\bar{\epsilon}}_{r}(\boldsymbol{r})$ is the relative permittivity dyad. The volume integral operator in (1) can be expressed as

$$
\mathcal{V}(\boldsymbol{F})(\boldsymbol{r})=\int_{\Omega} G_{0}\left(\boldsymbol{r}, \boldsymbol{r}^{\prime}\right) \boldsymbol{F}\left(\boldsymbol{r}^{\prime}\right) d V^{\prime},
$$

where $G_{0}$ is the Green's function of the background.

From representation (1) and the definition of the equivalent current (2), the volume integral equation formulation for the electric current (J-VIE) can be derived:

$$
\boldsymbol{J}^{i n c}=\boldsymbol{J}-\left(\overline{\bar{\epsilon}}_{r}-\overline{\bar{I}}\right) \cdot\left(\nabla \nabla+k_{0}^{2} \overline{\bar{I}}\right) \cdot \mathcal{V}(\boldsymbol{J}) .
$$

The incident current is defined as $\boldsymbol{J}^{i n c}=-i \omega \epsilon_{0}\left(\overline{\bar{\epsilon}}_{r}-\overline{\bar{I}}\right) \cdot \boldsymbol{E}^{i n c}$ The above integral equation can be solved in $L^{2}(\Omega)^{3}$ [14], [16].

\section{DisCRETIZATION}

Let us next consider the discretization of the volume integral equation (4). Since the unknown current $\boldsymbol{J}$ is discontinuous across material interfaces, basis functions should span $L^{2}(\Omega)$. We divide the volume of the object $\Omega$ with linear tetrahedral elements $k$, and define piecewise linear basis $\boldsymbol{b}_{k}^{i}$ and testing $\boldsymbol{t}_{k}^{i}$ 
functions on the tetrahedral mesh. The superindex $i$ denotes the $x$-, $y$ - or $z$ - component. The current is approximated as

$$
\begin{gathered}
\boldsymbol{J} \approx \sum_{k, i} c_{k}^{i} \boldsymbol{b}_{k}^{i}= \\
\sum_{k=1}^{N_{\text {tet }}} \sum_{p=1}^{4}\left(c_{k}^{x, p} N_{p} \hat{\boldsymbol{e}}_{x}+c_{k}^{y, p} N_{p} \hat{\boldsymbol{e}}_{y}+c_{k}^{z, p} N_{p} \hat{\boldsymbol{e}}_{z}\right) / \sqrt{V_{k}},
\end{gathered}
$$

where $\hat{\boldsymbol{e}}_{x}, \hat{\boldsymbol{e}}_{y}, \hat{\boldsymbol{e}}_{z}$ are the unit vectors in the Cartesian coordinate, $N_{p}$ are the standard linear nodal shape functions, and $V_{k}$ is the volume of tetrahedron $k$. Therefore, each tetrahedron contains 12 basis functions (four functions for each component). In addition, to obtain well-behaving discrete identity operator, which is independent on the mesh density, the basis and testing functions are scaled by $1 / \sqrt{V_{k}}$.

The method of moments is applied to convert the continuous equation into a discrete set of equations with the $L^{2}$-inner product defined as

$$
\langle\boldsymbol{F}, \boldsymbol{G}\rangle_{\Omega}=\int_{\Omega} \boldsymbol{F} \cdot \boldsymbol{G} \mathrm{d} r .
$$

It is worth noting that Galerkin's scheme in $L^{2}$ forces the interface conditions to be valid in a weak sense since from Helmholtz decomposition $\boldsymbol{F}=\nabla p+\nabla \times w, \in L^{2}$ we can see that

$$
\begin{gathered}
<\nabla p+\nabla \times \boldsymbol{w}, \boldsymbol{J}>=-<p, \nabla \cdot \boldsymbol{J}>+<\boldsymbol{w}, \nabla \times \boldsymbol{J}> \\
+<p, \boldsymbol{n} \cdot \boldsymbol{J}>+<\boldsymbol{w}, \boldsymbol{n} \times \boldsymbol{J}>,
\end{gathered}
$$

hence the function, its divergence, curl, and boundary values are properly tested. The functions in the decomposition are defined as $\boldsymbol{w} \in H_{\text {curl }}(\Omega)^{3}$ and $p \in H^{1}(\Omega)^{3}$.

Taking the inner product with the testing functions, using integration by parts, the elements of the discretized system matrix are

$$
\begin{gathered}
A_{m n}^{i j}=\int_{V_{m}} \boldsymbol{t}_{m}^{i} \cdot \boldsymbol{b}_{m}^{j} \mathrm{~d} V \\
+\int_{\partial V_{m}} \boldsymbol{n} \cdot\left(\overline{\bar{\tau}}^{T} \cdot \boldsymbol{t}_{m}^{i}\right) \cdot \int_{\partial V_{n}} G \boldsymbol{n}^{\prime} \cdot \boldsymbol{b}_{n}^{j} \mathrm{~d} S^{\prime} \mathrm{d} S \\
+\int_{V_{m}}\left(\nabla \cdot\left(\overline{\bar{\tau}}^{T} \cdot \boldsymbol{t}_{m}^{i}\right)\right) \int_{V_{n}} G \nabla^{\prime} \cdot \boldsymbol{b}_{n}^{j} \mathrm{~d} V^{\prime} \mathrm{d} V \\
-\int_{V_{m}}\left(\nabla \cdot\left(\overline{\bar{\tau}}^{T} \cdot \boldsymbol{t}_{m}^{i}\right)\right) \int_{\partial V_{n}} G \boldsymbol{n}^{\prime} \cdot \boldsymbol{b}_{n}^{j} \mathrm{~d} S^{\prime} \mathrm{d} V \\
-\int_{\partial V_{m}}^{\boldsymbol{n}} \cdot\left(\overline{\bar{\tau}}^{T} \cdot \boldsymbol{t}_{m}^{i}\right) \int_{V_{n}} G \nabla^{\prime} \cdot \boldsymbol{b}_{n}^{j} \mathrm{~d} V^{\prime} \mathrm{d} S \\
-\int_{V_{m}} \boldsymbol{t}_{m}^{i} \cdot \overline{\bar{\tau}} \cdot k_{0}^{2} \overline{\bar{I}} \cdot \int_{V_{n}} G \boldsymbol{b}_{n}^{j} \mathrm{~d} V^{\prime} \mathrm{d} V
\end{gathered}
$$

where $\overline{\bar{\tau}}=\left(\overline{\bar{\epsilon}}_{r}-\overline{\bar{I}}\right), V_{m}$ and $\partial V_{m}$ are the volume and the surface of tetrahedron $m$, respectively. The elements of the force vector read as

$$
b_{m}^{i}=\int_{V_{m}} \boldsymbol{t}_{m}^{i} \cdot \boldsymbol{J}^{i n c} \mathrm{~d} V .
$$

All the integrals in (8) are weakly singular, and are computed with the singularity extraction technique [18].

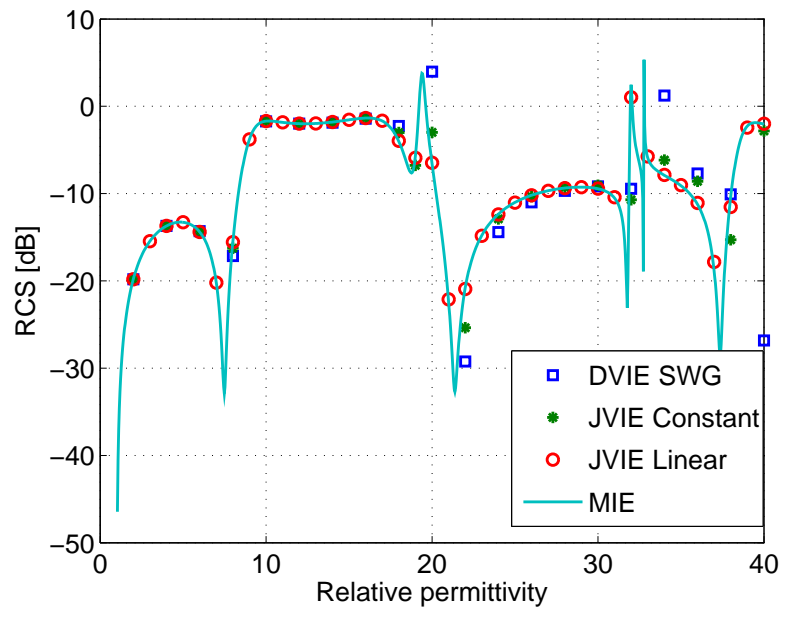

Fig. 1. Backscattered radar cross section as a function of permittivity of a homogeneous sphere with $k a=1$ computed by the J-VIE with the piecewise constant and linear basis functions.

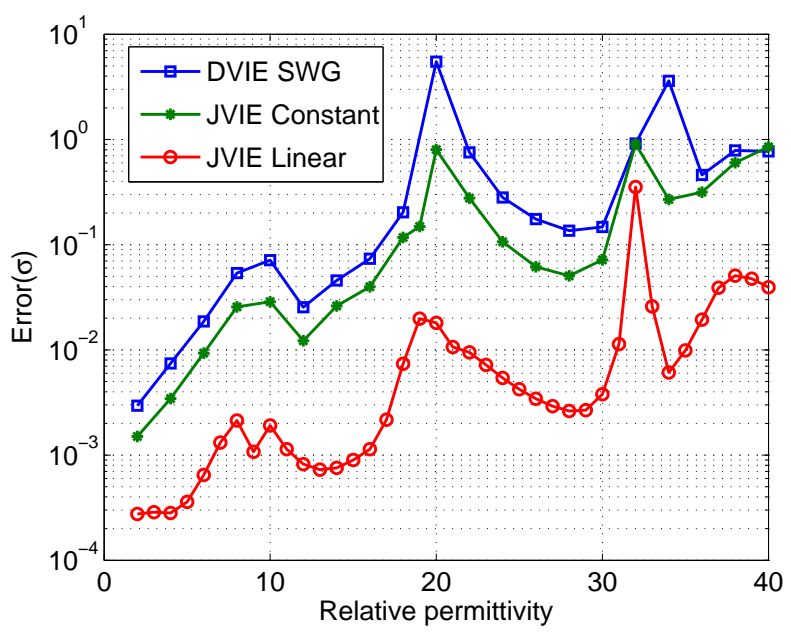

Fig. 2. Relative error of the far field scattering with respect to the permittivity of a homogeneous sphere with $k a=1$.

\section{NUMERICAL EXAMPLES}

In this section, we consider numerical examples and study accuracy of the J-VIE discretized with Galerkin's procedure with linear and constant functions. In the accuracy study, we use a far-field error quantity defined as follows:

$$
\operatorname{Error}(\sigma)=\sqrt{\frac{\int_{\gamma}(\sigma-\tilde{\sigma})^{2} \mathrm{~d} S}{\int_{\gamma} \tilde{\sigma}^{2} \mathrm{~d} S}},
$$

where $\gamma$ is the surface of unit sphere. The bistatic scattering cross section $\sigma / \tilde{\sigma}$ (calculated/exact) is given by

$$
\sigma(\theta, \phi)=\lim _{r \rightarrow \infty} 4 \pi r^{2} \frac{\left|\boldsymbol{E}^{s c a}(r, \theta, \phi)\right|^{2}}{\left|\boldsymbol{E}^{i n c}\right|^{2}} .
$$

To study the convergence of the actual solution $\boldsymbol{J}$, we need to define another error norm as follows

$$
\operatorname{Error}(\boldsymbol{J})=\sqrt{\frac{\int_{\Omega}\left(\boldsymbol{J} \cdot \boldsymbol{J}^{*}-\tilde{\boldsymbol{J}} \cdot \tilde{\boldsymbol{J}}^{*}\right)^{2} \mathrm{~d} V}{\int_{\Omega}\left(\tilde{\boldsymbol{J}} \cdot \tilde{\boldsymbol{J}}^{*}\right)^{2} \mathrm{~d} V}},
$$




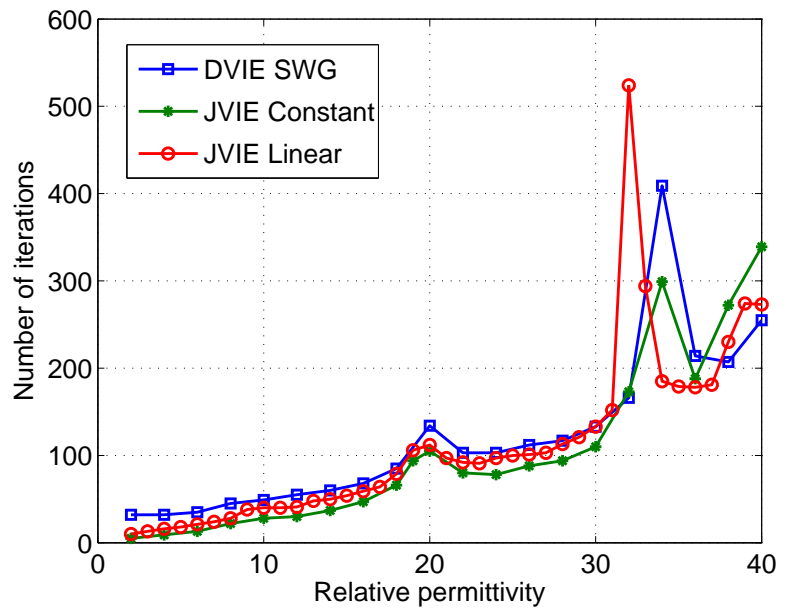

Fig. 3. Number of GMRES(200) iterations vs. relative permittivity of a sphere with $k a=1$.

where $\boldsymbol{J}$ is the numerical solution and $\tilde{\boldsymbol{J}}$ is the exact solution, and $\boldsymbol{J}^{*}$ stands for the complex conjugate of $\boldsymbol{J}$.

Let us first consider scattering by a dielectric sphere of size $k a=1$. Fig. 1 shows the radar cross section of the sphere in the backscattering direction as a function of permittivity, and the relative error of the scattered fields is plotted in Fig. 2. In this example, the sphere is discretized with 1624 tetrahedral elements giving 19488 unknowns when the linear basis functions are applied, and with piecewise constant functions, the number of elements is 6357 giving 19071 unknowns. Moreover, results computed by the commonly used DVIE formulation [1] with 6357 tetrahedra and 13152 SWG functions are included. Clearly, the discretization with piecewise linear basis functions gives much better accuracy with respect to the number of unknowns compared with the piecewise constant discretization.

To solve large problems, one needs to rely on iterative solvers and fast algorithms, e.g., the multilevel fast multipole algorithm MLFMA or the FFT-based acceleration techniques [19]-[22]. Hence, it is important to investigate the convergence of the iterative solution. The number of iterations required to solve the system as a function of permittivity of a sphere with $k a=1$ is plotted in Fig. 3. The GMRES(200) solver is used with the tolerance $t o l=10^{-5}$. The iteration count with the linear basis functions is almost the same as the iteration count with the constant functions. Peaks in Fig. 3 are due to Mie resonances, however, the lowest order functions cannot catch all of them properly since the element size is too large at high permittivities. In addition, we have observed that the iteration counts are independent of the mesh density assuming that the elements are small enough. Although, the iteration count depends on the permittivity since the spectrum of the integral operator is also permittivity dependent [16].

The discretization with the piecewise constant approximations provides a convergence rate of $\mathcal{O}(h)$ for the internal field, and $\mathcal{O}\left(h^{2}\right)$ for the far-field where $h$ is the average edgelength. As demonstrated in Fig. 4, with fully linear approximations the convergence rate in the near field is of order $\mathcal{O}\left(h^{2}\right)$. Fig.

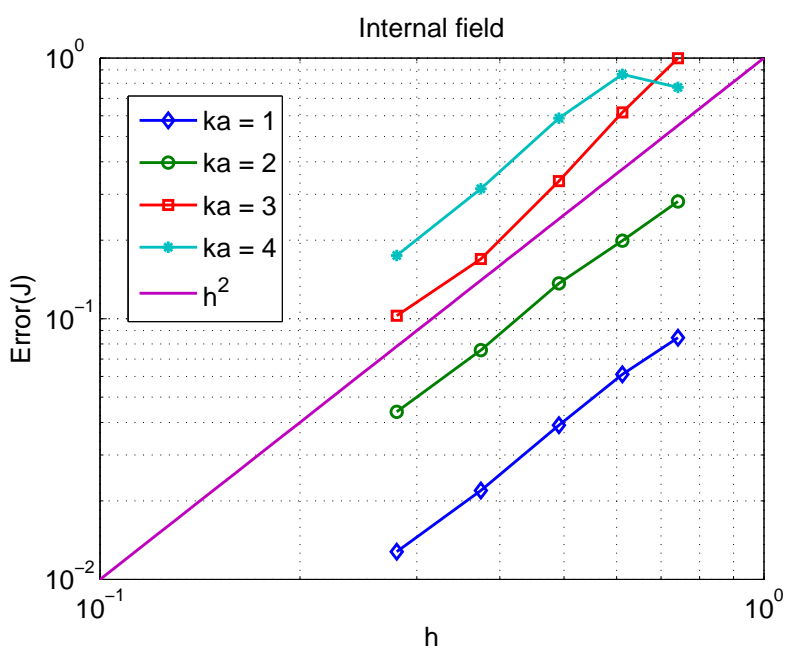

Fig. 4. Relative error of the solutions (12) as functions of averaged edge lengths for dielectric spheres $\left(\epsilon_{r}=5\right)$ of different sizes. Only the results with the linear basis function are plotted.

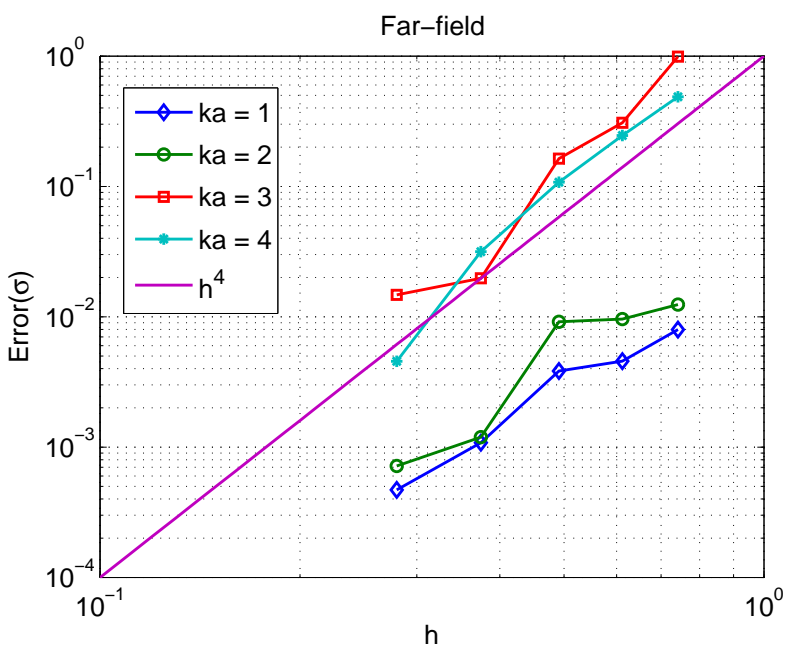

Fig. 5. Convergence of the scattering cross sections (10) computed by using piecewise linear functions in the same case as in Fig. 4.

5 shows that in the far field, the convergence rate with the linear functions approximately follows the trend $\mathcal{O}\left(h^{4}\right)$. With both approximations, linear and constant, the convergence rate in the far field is faster than in the near field since the operator used for computing the far-fields from the solution is a smoothing operator.

Finally, we will take a look at the internal electric field of an inhomogeneous cube with edgelength $l=1 \mathrm{~m}$ whose center is at the origin. The permittivity $\epsilon_{r}=4$ if $x<0$ and $\epsilon_{r}=8$ if $x>0$. The incident wave is linearly polarized along $\mathrm{x}$-axis and it is propagating along z-axis. Each component of the real part of the electric field along X-axis is plotted in Figs. 6 and 7 computed by the J-VIE with the piecewise linear and constant expansion functions, respectively. The reference result is computed by the surface integral equation method [23].

The cube is discretized with 7238/1034 elements giving 21714/12408 DoFs when the constant/linear functions are applied. The internal fields are directly retrieved from the 


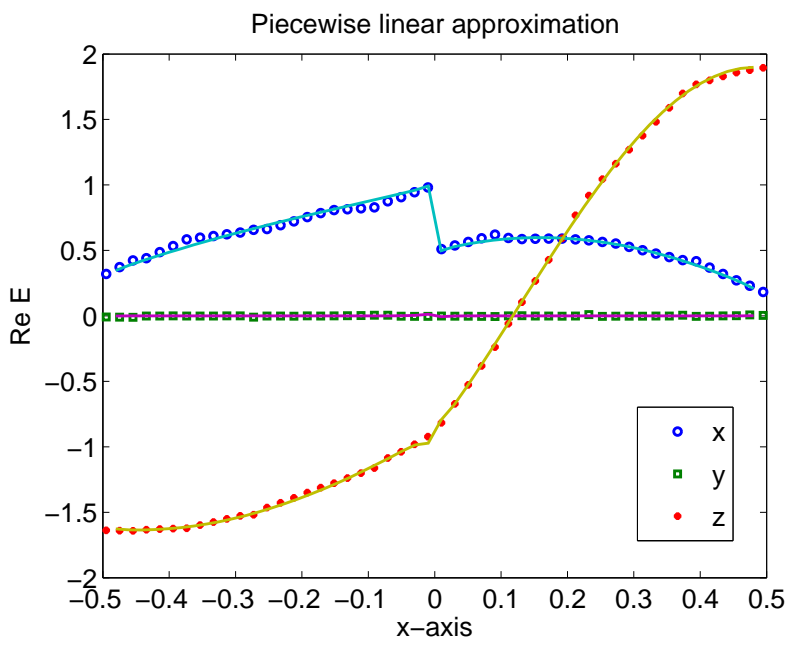

Fig. 6. The real part of the internal electric field (x-,y-, and z-components) of the inhomogeneous cube plotted along x-axis. The permittivity is $\epsilon_{r}=4$ when $x<0$ and $\epsilon_{r}=8$ when $x>0$. The reference (solid lines) is computed by the surface integral equation method.

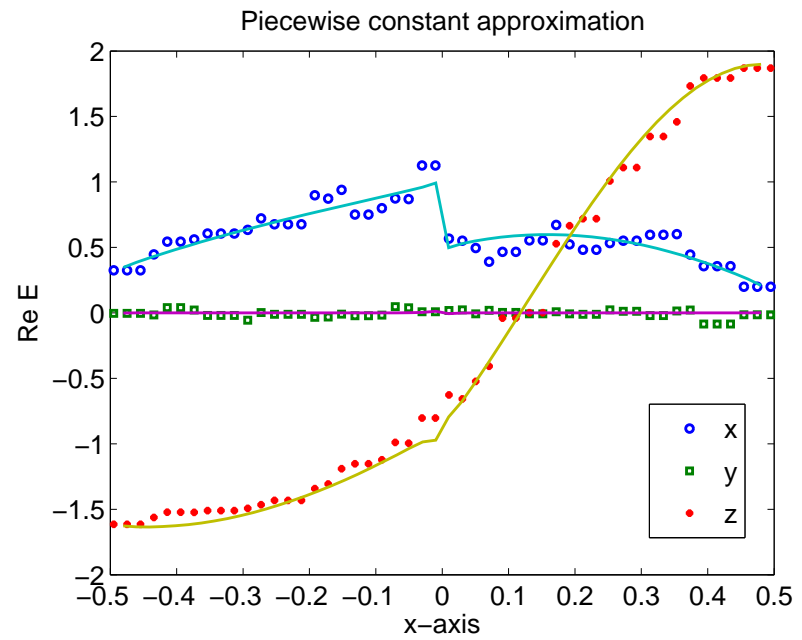

Fig. 7. As in Fig.6 computed with piecewise constant functions.

solution $\left(\boldsymbol{E}=\frac{i}{\omega \epsilon_{0}}\left(\overline{\bar{\epsilon}}_{r}-\overline{\bar{I}}\right)^{-1} \cdot \boldsymbol{J}\right)$, hence it is not suprising that the higher order expansion gives more accurate representation for the internal fields.

\section{Conclusions}

The volume integral equation for the electric current has been discretized in $L^{2}$ by using tetrahedral elements and piecewise linear approximations. The numerical examples show that the discretization with the piecewise linear basis and testing functions provides much better accuracy compared to the discetization utilizing the piecewise constant approximations. The convergence rate of the solution follows the trend $\mathcal{O}\left(h^{2}\right)$ for the equivalent current and $\mathcal{O}\left(h^{4}\right)$ for the far fields assuming that the geometric error is insignificant.

\section{REFERENCES}

[1] D. Schaubert, D. Wilton and A. Glisson, "A tetrahedral modeling method for electromagnetic scattering by arbitrarily inhomogeneous dielectric bodies", IEEE Trans. Ant. and Propag., vol. 32, no. 1, 77-85, 1984.
[2] M. M. Botha, "Solving the volume integral equations of electromagnetic scattering", J. Comput. Phys., vol. 218, pp. 141-158, 2006.

[3] S. Antenor de Carvalho, and L. de Souza Mendes, "Scattering of EM waves by inhomogeneous dielectrics with the use of the method of moments and 3-D solenoidal basis functions", Microw. Opt. Technol. Lett., vol. 23, pp. 42-46, Oct 1999.

[4] M.-K. Li and W. C. Chew, "Applying divergence-free condition in solving the volume integral equation", Progress in Electromagnetic Research, PIER 57, pp. 311-333, 2006.

[5] G. Rubinacci and A. Tamburrino, "A broadband volume integral formulation based on edge-elements for full-wave analysis of lossy interconnects",IEEE Trans. Ant. and Propag., vol. 54, no. 10, pp. 2977-2989, Oct. 2006.

[6] L. E. Sun and W. C. Chew, "A novel formulation of the volume integral equation for electromagnetic scattering", Waves in Random and Complex Media, vol. 19, no. 1, pp. 162-180, Feb. 2009.

[7] J. Markkanen, C-C. Lu, X. Cao, and P. Ylä-Oijala, "Analysis of volume integral equation formulations for scattering by high-contrast penetrable objects", IEEE Trans. Ant. and Propag., vol. 60, no. 5, pp. 2367-2374, May 2012.

[8] C.-C. Lu, P. Ylä-Oijala, M. Taskinen and J. Sarvas, "Comparison of two volume integral equation formulations for solving electromagnetic scattering by inhomogeneous dielectric objects", International IEEE APS Symposium 2009, Charleston, USA.

[9] M. I. Sancer, K. Sertel, J. L. Volakis and P. Van Alstine, "On volume integral equations", IEEE Trans. Ant. and Propag., vol. 54, no. 5, pp. 1488-1495, May, 2006

[10] N. A. Ozdemir and Jin-Fa Lee, "A nonconformal volume integral equation for electromagnetic scattering from penetrable objects", IEEE Trans. Magnetics, vol. 43, no. 4, pp. 1369-1372, Apr. 2007.

[11] J. Markkanen, P. Ylä-Oijala, and A. Sihvola, "Discretization of volume integral equation formulations for extremely anisotropic materials", IEEE Trans. Ant. and Propag., vol. 60, no. 11, pp. 5195-5202, Nov. 2012.

[12] P. De Doncker, "A potential integral equations method for electromagnetic scattering by penetrable bodies", IEEE Trans. Ant. and Propag., vol. 49, no. 7, pp. 1037-1042, July, 2001

[13] R. Chang, and V. Lomakin, "Potential-based volume integral equations", 2011 IEEE International Symposium on Antennas and Propagation (APSURSI), pp. 2712-2715, 3-8 July 2011.

[14] M. C. van Beurden and S. J. L. van Eijndhoven, "Gaps in present discretization schemes for domain integral equations", International Conference on Electromagnetics in Advanced Applications, ICEAA 2007, Torino.

[15] W. C. Chew, J.-M. Jin, E. Michielssen and J. Song, Fast and efficient algorithms in computational electromagnetics, Artech House, Boston, 2001.

[16] M. Costabel, E. Darrigrand, and H. Sakly, "The essential spectrum of the volume integral operator in electromagnetic scattering by a homogeneous body", Comptes Rendus Mathematique, vol. 350, no. 3-4. pp. 193-197, Feb. 2012.

[17] M. C. van Beurden and S. J. L. van Eijndhoven, Well-posedness of domain integral equations for a dielectric object in homogeneous background, J. Eng. Math(2008) 62:289-302.

[18] S. Järvenpää, M. Taskinen, and P. Ylä-Oijala, "Singularity extraction technique for integral equation methods with higher order basis functions on plane triangles and tetrahedra", J. Numer. Meth. Engng, vol. 58, pp. $1149-1165,2003$.

[19] V. Rokhlin, "Rapid solution of integral equations of scattering theory in two dimensions", J. Comput. Phys., vol. 86, no. 2, pp. 414-439, Feb. 1990.

[20] C.-C. Lu, "A fast algorithm based on volume integral equation for analysis of arbitrarily shaped dielectric radomes", IEEE Trans. Ant. and Propag., vol. 51, no. 3, pp. 606-612, Mar. 2003.

[21] E. Bleszynski, M. Bleszynski, and T. Jaroszewicz, "AIM: Adaptive integral method for solving large-scale electromagnetic scattering and radiation problems", Radio Science, vol. 31, no. 5, pp. 1225-1251, Sept. 1996.

[22] J.R. Phillips, and J.K. White, "A precorrected-FFT method for electrostatic analysis of complicated 3-D structures", IEEE Trans. ComputerAided Design of Integrated Circuits and Systems, vol. 16, no. 10, pp. 1059-1072, Oct. 1997.

[23] P. Ylä-Oijala, M. Taskinen, and J. Sarvas, "Surface integral equation method for general composite metallic and dielectric structures with junctions", Progress in Electromagnetic Research, PIER 52, pp. 81-108, 2005. 